\title{
Fractional Diffusion Equations for Lattice and Continuum: Grünwald-Letnikov Differences and Derivatives Approach
}

\author{
Vasily E. Tarasov \\ Skobeltsyn Institute of Nuclear Physics, Lomonosov Moscow State University, Moscow 119991, Russia \\ Correspondence should be addressed to Vasily E. Tarasov; tarasov@theory.sinp.msu.ru
}

Received 30 August 2014; Accepted 24 November 2014; Published 8 December 2014

Academic Editor: Adam Lipowski

Copyright (C) 2014 Vasily E. Tarasov. This is an open access article distributed under the Creative Commons Attribution License, which permits unrestricted use, distribution, and reproduction in any medium, provided the original work is properly cited.

\begin{abstract}
Fractional diffusion equations for three-dimensional lattice models based on fractional-order differences of the Grünwald-Letnikov type are suggested. These lattice fractional diffusion equations contain difference operators that describe long-range jumps from one lattice site to another. In continuum limit, the suggested lattice diffusion equations with noninteger order differences give the diffusion equations with the Grünwald-Letnikov fractional derivatives for continuum. We propose a consistent derivation of the fractional diffusion equation with the fractional derivatives of Grünwald-Letnikov type. The suggested lattice diffusion equations can be considered as a new microstructural basis of space-fractional diffusion in nonlocal media.
\end{abstract}

\section{Introduction}

Fractional calculus and differential equation of noninteger orders [1-5] have a long history that is connected with the names of famous scientists such as Liouville, Riemann, Grünwald, Letnikov, Marchaud, and Riesz. Derivatives and integrals of noninteger orders have a lot of applications in different areas of physics [6-10]. Fractional calculus is a powerful tool to describe processes in continuously distributed media with nonlocal properties. As it was shown in $[11,12]$, the continuum equations with fractional derivatives are directly connected [7] to lattice models with long-range interactions. The lattice equations for fractional nonlocal media and the correspondent continuum equations have been considered recently in [13-15]. Fractional-order differences and the correspondent derivatives have been first proposed by Grünwald [16] and by Letnikov [17]. At the present time these generalized differences and derivatives are called the Grünwald-Letnikov fractional differences and derivatives $[1-3,18]$. One-dimensional lattice models with long-range interactions of the Grünwald-Letnikov type and the correspondent fractional differential and integral continuum equations have been suggested in [19]. The suggested form of long-range interaction is based on the form of the left-sided and right-sided Grünwald-Letnikov fractional differences. A possible form of lattice vectors calculus based on the fractional-order differences of the Grünwald-Letnikov type has been suggested in [20]. In this paper, we apply this approach to describe diffusion on lattices with longrange jumps and to derive fractional diffusion equations for nonlocal continuum with power-law nonlocality.

The diffusion equations describe the change of probability of a random function in space and time in transport processes, and they usually have the form of second-order partial differential equation of parabolic type. Unfortunately, for complex nonlocal media, the usual second-order diffusion equation cannot adequately describe real processes. For example, the diffusion processes with the Poissonian waiting time and the Lévy distribution for the jump length cannot be described by equation with second-order derivatives with respect to coordinates. The Lévy distribution describes the Lévy flights $[21,22]$ that are random walks, where the jump lengths have probability distributions with heavy tails. The Lévy motion can be described by equation with spatial derivatives of noninteger orders $\mu$, [22]. In this case, the moment of order $\delta$ for the Lévy motion has the form $\left\langle|x(t)|^{\delta}\right\rangle \sim t^{\delta / \mu}$, where $0<\delta<\mu \leq 2$. Usually the spacefractional diffusion equations are obtained from the secondorder differential equations by replacing the second-order space derivatives by fractional-order derivatives. Fractional diffusion equations with coordinate derivatives of noninteger order have been suggested in [23]. The solutions and 
properties of these equations are considered in [24-26]. The diffusion equations with fractional coordinate derivatives were also considered in $[8,9,27]$.

In this paper we propose a consistent derivation of the space-fractional diffusion equation with Grünwald-Letnikov derivatives of noninteger orders by using lattice models with long-range jumps that is considered new microstructural basis to describe fractional diffusion processes in nonlocal media. In this paper, we suggest a lattice equation for probability density of particle in unbounded homogeneous three-dimensional lattice with long-range jumps between lattice sites. We prove that continuous limit for the suggested lattice diffusion equation gives the space-fractional diffusion equation for nonlocal continuum. The fractional differential equation for continuum contains the Grünwald-Letnikov derivatives on noninteger orders. Continuum models can be considered as a continuous limit of lattice models, where the length-scales of a continuum element are much larger than the distances between the lattice sites. The suggested approach to derive the space-fractional diffusion equations can serve as a microstructural basis to describe the spatialfractional diffusion processes.

\section{Fractional Diffusion Equation for Lattice}

The differences of fractional order and the correspondent fractional derivatives have been introduced by Grünwald in 1867 and independently by Letnikov in 1868 .

Differences of noninteger orders are defined as a generalization of the integer order difference

$$
\nabla_{a, \pm}^{m} f(x)=\sum_{n=1}^{m} \frac{(-1)^{n} m !}{n !(m-n) !} f(x \mp n a), \quad\left(a \in \mathbb{R}_{+}, m \in \mathbb{N}\right) .
$$

The Fourier transformation $F$ of the fractional-order differences of Grünwald-Letnikov type (see Section 20 of $[1,2]$ ) has the form

$$
\mathscr{F}\left\{\nabla_{a, \pm}^{\alpha} f(x)\right\}(k)=\left(1-e^{ \pm a k}\right)^{\alpha} \mathscr{F}\{f\}(k) .
$$

The differences of integer orders (1) are defined by the finite series. The differences of noninteger order $\alpha \in \mathbb{R}_{+}$are defined as infinite series (see Section 20 of $[1,2]$ ). Fractional-order differences of Grünwald-Letnikov type are defined by

$$
\nabla_{a, \pm}^{\alpha} f(x)=\sum_{n=0}^{\infty} \frac{(-1)^{n} \Gamma(\alpha+1)}{\Gamma(n+1) \Gamma(\alpha-n+1)} f(x \mp n a), \quad(a>0) .
$$

The difference $\nabla_{a++}^{\alpha}$ is called left-sided fractional difference, and $\nabla_{a,-}^{\alpha}$ is called the right-sided fractional difference. We note that the series in (3) converges absolutely and uniformly for every bounded function $f(x)$ and $\alpha>0$.

Using the fractional-order differences (3), we can consider the derivatives of noninteger orders. The left- and rightsided Grünwald-Letnikov derivatives of fractional order $\alpha>$ 0 are defined by

$$
{ }^{\mathrm{GL}} D_{x, \pm}^{\alpha} f(x)=\lim _{a \rightarrow 0+} \frac{\nabla_{a, \pm}^{\alpha} f(x)}{|a|^{\alpha}} .
$$

For integer values of $\alpha=m \in \mathbb{N}$ the Grünwald-Letnikov derivatives (4) are equal to the usual integer order derivatives up to the sign in the form

$$
{ }^{\mathrm{GL}} D_{x, \pm}^{m} f(x)=( \pm 1)^{m} \frac{d^{m} f(x)}{d x^{m}} .
$$

We can note that the Grünwald-Letnikov fractional derivatives coincide with the Marchaud fractional derivatives for the functions from $L_{r}(\mathbb{R})$, where $1 \leqslant r<\infty$ (see Theorem 20.4 in $[1,2])$.

Let us consider three-dimensional unbounded and bounded lattices. Physical lattices are characterized by space periodicity. For unbounded lattices we can use three noncoplanar vectors $\mathbf{a}_{1}, \mathbf{a}_{2}$, and $\mathbf{a}_{2}$ that are the shortest vectors by which a lattice can be displaced and be brought back into itself. Sites of this lattice can be characterized by the number vector $\mathbf{n}=\left(n_{1}, n_{2}, n_{3}\right)$, where $n_{j}(j=1,2,3)$ are integer. For simplification, we consider a lattice with mutually perpendicular primitive lattice vectors $\mathbf{a}_{j}(j=1,2,3)$. We choose directions of the axes of the Cartesian coordinate system that coincide with the vector $\mathbf{a}_{j}$. In this case $\mathbf{a}_{j}=$ $a_{j} \mathbf{e}_{j}$, where $a_{j}=\left|\mathbf{a}_{j}\right|>0$ and $\mathbf{e}_{j}$ are the basis vectors of the Cartesian coordinate system. This means that we use a primitive orthorhombic Bravais lattice. Then the vector $\mathbf{n}$ can be represented as $\mathbf{n}=n_{1} \mathbf{e}_{1}+n_{2} \mathbf{e}_{2}+n_{3} \mathbf{e}_{3}$.

Choosing a coordinate origin at one of the lattice sites, the positions of all other sites with $\mathbf{n}=\left(n_{1}, n_{2}, n_{3}\right)$ are described by the vector $\mathbf{r}(\mathbf{n})=n_{1} \mathbf{a}_{1}+n_{2} \mathbf{a}_{2}+n_{3} \mathbf{a}_{3}$. The lattice sites are numbered by $\mathbf{n}$, so that vector $\mathbf{n}$ can be considered as a number vector of the corresponding particle. We assume that the positions of particles in the lattice coincide with the lattice sites. The distribution function, which describes probability density for lattice site $\mathbf{n}$, will be denoted by $f(\mathbf{n}, t)=f\left(n_{1}, n_{2}, n_{3}, t\right)$. This function satisfies the conditions

$$
\begin{array}{r}
\sum_{n_{1}=-\infty}^{+\infty} \sum_{n_{2}=-\infty}^{+\infty} \sum_{n_{3}=-\infty}^{+\infty} f\left(n_{1}, n_{2}, n_{3}, t\right)=1, \\
f\left(n_{1}, n_{2}, n_{3}, t\right) \geq 0 \quad(t \geq 0) .
\end{array}
$$

To describe dynamics of the distribution function $f(\mathbf{n}, t)$ in the lattice models with long-range jumps between sites, we define fractional-order difference operators of the GrünwaldLetnikov type in the direction $\mathbf{e}_{j}=\mathbf{a}_{j} /\left|\mathbf{a}_{j}\right|$ of the lattice. Fractional-order difference operators of the GrünwaldLetnikov type for unbounded lattice are the operators $\left.\mathrm{GL}^{ \pm} \mathbb{D}_{L}^{ \pm} \begin{array}{c}\alpha_{j} \\ j\end{array}\right]$ that act on the function $f(\mathbf{m}, t)$ as

$$
\begin{array}{r}
\mathrm{GL}_{\mathbb{D}_{L}}^{ \pm}\left[\begin{array}{c}
\alpha_{j} \\
j
\end{array}\right] f(\mathbf{m}, t)=\frac{1}{a_{j}^{\alpha}} \sum_{m_{j}=-\infty}^{+\infty}{ }^{\mathrm{GL}} K_{\alpha_{j}}^{ \pm}\left(n_{j}-m_{j}\right) f(\mathbf{m}, t) \\
\left(\alpha_{j}>0, j=1,2,3\right),
\end{array}
$$

where the kernels ${ }^{\mathrm{GL}} K_{\alpha}^{ \pm}(n)$ are defined by the equation

$$
\begin{array}{r}
{ }^{\mathrm{GL}} K_{\alpha_{j}}^{ \pm}(n)=\frac{(-1)^{n} \Gamma\left(1+\alpha_{j}\right)(H[n] \pm H[-n])}{2 \Gamma(|n|+1) \Gamma\left(1+\alpha_{j}-|n|\right)}, \\
\left(\alpha_{j}>0, n \in \mathbb{Z}\right),
\end{array}
$$


and $\Gamma(z)$ is the gamma function, $H[n]$ is the discrete variable Heaviside step function that is defined as $H[n]=1$ for $n \geq 0$, and $H[n]=0$ for $n<0$, where $n \in \mathbb{Z}$. The parameter $\alpha_{j}$ is called the order of the operator. It should be noted that the definition of $H[0]=1$ for discrete variable Heaviside function is significant for us, since it allows us to write the kernels ${ }^{\mathrm{GL}} K_{\alpha}^{+}(n)$ in the simple form without allocating repeated zero terms. Fractional-order difference operators (7) can be called a lattice fractional partial derivative in the direction $\mathbf{e}_{j}=\mathbf{a}_{j} /\left|\mathbf{a}_{j}\right|$.

It should be noted that one-dimensional lattice models with the long-range interaction of the form ${ }^{\mathrm{GL}} K_{\alpha}^{+}(n)$ and correspondent fractional nonlocal continuum models have been suggested in [19]. The lattice operators (7) recently have been proposed in [20].

It is easy to see that kernels ${ }^{\mathrm{GL}} K_{\alpha}^{+}(n)$ and ${ }^{\mathrm{GL}} K_{\alpha}^{-}(n)$ are even and odd functions such that ${ }^{\mathrm{GL}} K_{\alpha}^{ \pm}(-n)= \pm{ }^{\mathrm{GL}} K_{\alpha}^{ \pm}(n)$. The form of the lattice operators (7) can be defined by the addition and subtraction of the Grünwald-Letnikov fractional differences

$$
\begin{aligned}
& \mathrm{GL}_{\mathbb{D}_{L}^{ \pm}}\left[\begin{array}{c}
\alpha \\
j
\end{array}\right] f(\mathbf{m}, t) \\
&=\frac{1}{\left|a_{j}\right|^{\alpha}} \sum_{m_{j}=0}^{\infty} \frac{(-1)^{m_{j}} \Gamma(1+\alpha)}{2 \Gamma\left(m_{j}+1\right) \Gamma\left(1+\alpha-m_{j}\right)} \\
& \quad \times\left(f\left(\mathbf{n}-m_{j} \mathbf{e}_{j}, t\right) \pm f\left(\mathbf{n}-m_{j} \mathbf{e}_{j}, t\right)\right) .
\end{aligned}
$$

We should note that in (9) the summation is realized over nonnegative values $m_{j}$, in contrast to the sum over all integer values in (7).

For bounded physical lattice models the fractional-order difference operators also can be defined. Fractional-order difference operators of the Grünwald-Letnikov type for bounded lattice with $m_{j}^{1} \leq m_{j} \leq m_{j}^{2}$ are the operators ${ }_{B}^{\mathrm{GL}} \mathbb{D}_{L}^{ \pm}\left[\begin{array}{c}\alpha_{j} \\ j\end{array}\right]$ that act on the function $f(\mathbf{m}, t)$ as

$$
\begin{array}{r}
{ }_{B}^{\mathrm{GL}} \mathbb{D}_{L}^{ \pm}\left[\begin{array}{c}
\alpha_{j} \\
j
\end{array}\right] f(\mathbf{m}, t)=\frac{1}{a_{j}^{\alpha}} \sum_{m_{j}=m_{j}^{1}}^{m_{j}^{2}}{ }^{\mathrm{GL}} K_{\alpha_{j}}^{ \pm}\left(n_{j}-m_{j}\right) f(\mathbf{m}, t) \\
(j=1,2,3),
\end{array}
$$

where kernels ${ }^{\mathrm{GL}} K_{\alpha_{j}}^{ \pm}(n)$ are defined by (8). The suggested forms of fractional difference operators for bounded physical lattice models are based on the Grünwald-Letnikov fractional differences on finite intervals (see Section 20.4 of $[1,2]$ ). For the finite interval $\left[x_{j}^{1}, x_{j}^{2}\right]$, the integer values $m_{j}^{1}, m_{j}^{2}$, and $m_{j}$ are defined by the equations

$$
m_{j}^{1}=\left[\frac{x_{j}^{1}}{a_{j}}\right], \quad m_{j}^{2}=\left[\frac{x_{j}^{2}}{a_{j}}\right], \quad m_{j}=\left[\frac{x_{j}}{a_{j}}\right],
$$

where the brackets [.] of (11) mean the floor function that maps a real number to the largest previous integer number.
Using the semigroup property for fractional differences of nonnegative orders (see Property 2.29 in [3]), it is easy to show that the semigroup property holds for the fractional operators (7) in the form

$$
\mathrm{GL}_{\mathbb{D}_{L}^{ \pm}}^{ \pm}\left[\begin{array}{c}
\alpha \\
j
\end{array}\right]{ }^{\mathrm{GL}} \mathbb{D}_{L}^{ \pm}\left[\begin{array}{c}
\beta \\
j
\end{array}\right]={ }^{\mathrm{GL}} \mathbb{D}_{L}^{ \pm}\left[\begin{array}{c}
\alpha+\beta \\
j
\end{array}\right], \quad(\alpha>0, \beta>0) .
$$

Using this equation, it is easy to prove the commutativity and the associativity of the lattice operator (7) of the GrünwaldLetnikov type. The commutativity and associativity of the fractional operators (7) of the Grünwald-Letnikov type for different directions are obvious.

To describe isotropic physical lattices we should use the difference operators ${ }^{\mathrm{GL}} \mathbb{D}_{L}^{ \pm}\left[\begin{array}{c}\alpha_{j} \\ j\end{array}\right]$ and ${ }_{B}^{\mathrm{GL}} \mathbb{D}_{L}^{ \pm}\left[\begin{array}{c}\alpha_{j} \\ j\end{array}\right]$ with orders $\alpha_{j}=\alpha$ for all $j=1,2,3$.

Let us give possible equations for distribution function $f(\mathbf{n}, t)$ on unbounded and bounded lattices. For unbounded homogeneous lattice the diffusion equation for probability density can be considered in the form

$$
\begin{aligned}
\frac{\partial f(\mathbf{n}, t)}{\partial t}= & -\sum_{i=1}^{3} g_{i}^{\mathrm{GL}} \mathbb{D}^{ \pm}\left[\begin{array}{c}
\alpha_{i} \\
i
\end{array}\right] f(\mathbf{m}, t) \\
& +\sum_{i, j=1}^{3} g_{i j}{ }^{\mathrm{GL}} \mathbb{D}^{ \pm, \pm}\left[\begin{array}{cc}
\alpha_{i} & \beta_{j} \\
i & j
\end{array}\right] f(\mathbf{m}, t) .
\end{aligned}
$$

For bounded lattice we should use the fractional difference operators (10), and the correspondent analog of (13) has the form

$$
\begin{aligned}
\frac{\partial f(\mathbf{n}, t)}{\partial t}= & -\sum_{i=1}^{3} g_{i}{ }_{B} \mathbb{D}^{ \pm}\left[\begin{array}{c}
\alpha_{i} \\
i
\end{array}\right] f(\mathbf{m}, t) \\
& +\sum_{i, j=1}^{3} g_{i j B}{ }^{\mathrm{GL}} \mathbb{D}^{ \pm, \pm}\left[\begin{array}{cc}
\alpha_{i} & \beta_{j} \\
i & j
\end{array}\right] f(\mathbf{m}, t) .
\end{aligned}
$$

Equations (13) and (14) are the three-dimensional lattice diffusion equations that describe fractional diffusion processes with the lattice jumps. Here $f(\mathbf{n}, t)$ is the probability density function to find the test particle at site $\mathbf{n}$ at time $t$. The italics $i, j \in\{1 ; 2 ; 3\}$ are the coordinate indices, and $g_{i}$ and $g_{i j}$ are lattice coupling constants.

The first and second terms of the right-hand side of (13) and (14) describe the particle drift and diffusion on the lattice. These correspondent kernels describe the longrange drift and diffusion to $\mathbf{n}$-site from all other $\mathbf{m}$-sites. Parameters $\alpha_{i}$ and $\beta_{j}$ in the kernels are positive real numbers that characterize how quickly the intensity of the drift and diffusion processes in the lattice decrease with increasing the value $\mathbf{n}-\mathbf{m}$. Kernels $K_{\alpha_{j}}^{ \pm}\left(n_{j}-m_{j}\right)$, where $j=1,2,3$, describe long-range jumps in direction $\mathbf{a}_{j}$ with lattice step length $\left|n_{j}-m_{j}\right|$ in the lattice. In (13), we use the combination of the lattice operators

$$
\mathrm{GL}_{\mathbb{D}^{ \pm, \pm}}\left[\begin{array}{cc}
\alpha_{i} & \beta_{j} \\
i & j
\end{array}\right]={ }^{\mathrm{GL}} \mathbb{D}^{ \pm}\left[\begin{array}{c}
\alpha_{i} \\
i
\end{array}\right] \mathrm{GL}^{ \pm}\left[\begin{array}{c}
\beta_{j} \\
j
\end{array}\right]
$$


where $i, j$ take values from the set $\{1 ; 2 ; 3\}$. The action of the operator (15) on the lattice probability density $f(\mathbf{m}, t)$ is

$$
\begin{aligned}
\mathrm{GL}_{\mathbb{D}^{ \pm, \pm}}^{ \pm} & {\left[\begin{array}{cc}
\alpha_{i} & \beta_{j} \\
i & j
\end{array}\right] f(\mathbf{m}, t) } \\
& =\sum_{m_{i}=-\infty}^{+\infty} \sum_{m_{j}=-\infty}^{+\infty} K_{\alpha_{i}}^{ \pm}\left(n_{i}-m_{i}\right) K_{\beta_{j}}^{ \pm}\left(n_{j}-m_{j}\right) f(\mathbf{m}, t) .
\end{aligned}
$$

Equations (13) and (14) describe fractional diffusion processes on the physical lattices, where long-range jumps can be realized. The lattice diffusion equations (13) and (14) can be considered as lattice analogs of the fractional diffusion equations for the processes with the Poissonian waiting time and the Lévy distribution for the jump length.

Suggested lattice equations (13) and (14) can be considered as master equations that allow us to describe timeevolution of particles and quasiparticles on lattice since evolution is modelled as being in exactly one of the countable numbers of lattice sites at any given time, where switching between sites is treated probabilistically. These equations are differential equations for the variation over time of the probabilities that the particle occupies each of the lattice sites.

\section{Fractional Diffusion Equation for Continuum}

To describe fractional diffusion in the nonlocal continua, we should use fractional derivatives with respect to space coordinates instead of the lattice operators. Continuum analogs of the fractional-order difference operators of the GrünwaldLetnikov type are the fractional derivatives of GrünwaldLetnikov type.

Fractional-order difference operators $\mathrm{GL}_{\mathbb{D}_{L}^{ \pm}}\left[\begin{array}{c}\alpha_{j} \\ j\end{array}\right]$ defined by (7) are transformed by the continuous limit operation into the fractional derivative of Grünwald-Letnikov type with respect to coordinate $x_{j}$ in the form

$$
\lim _{a_{j} \rightarrow 0+}\left({ }^{\mathrm{GL}} \mathbb{D}_{L}^{ \pm}\left[\begin{array}{c}
\alpha_{j} \\
j
\end{array}\right] f(\mathbf{m}, t)\right)={ }^{\mathrm{GL}} \mathbb{D}_{C}^{ \pm}\left[\begin{array}{c}
\alpha_{j} \\
j
\end{array}\right] f(\mathbf{r}, t),
$$

where ${ }^{\mathrm{GL}} \mathbb{D}_{C}^{ \pm}\left[\begin{array}{c}\alpha_{j} \\ j\end{array}\right]$ are the continuum fractional derivatives of the Grünwald-Letnikov type that are defined by

$$
\mathrm{GL}_{\mathbb{D}_{C}^{ \pm}}^{ \pm}\left[\begin{array}{c}
\alpha \\
j
\end{array}\right]=\frac{1}{2}\left({ }^{\mathrm{GL}} D_{x_{j},+}^{\alpha}{ }^{\mathrm{GL}} D_{x_{j},-}^{\alpha}\right),
$$

which contain the Grünwald-Letnikov fractional derivatives ${ }^{\mathrm{GL}} D_{x_{j}, \pm}^{\alpha}$ with respect to space coordinate $x_{j}$ that can be written as

$$
\begin{aligned}
{ }^{\mathrm{GL}} D_{x_{j}, \pm}^{\alpha} f(\mathbf{r}, t)=\lim _{a_{j} \rightarrow 0+} \frac{1}{\left|a_{j}\right|^{\alpha}} \sum_{m_{j}=0}^{\infty} & \frac{(-1)^{m_{j}} \Gamma(\alpha+1)}{\Gamma\left(m_{j}+1\right) \Gamma\left(\alpha-m_{j}+1\right)} \\
& \times f\left(\mathbf{r} \mp m_{j} \mathbf{a}_{j}, t\right), \quad(\alpha>0) .
\end{aligned}
$$

This statement can be proved by analogy with the proof for lattice model with long-range interaction of the GrünwaldLetnikov type suggested in [19].

It is important to note that the Grünwald-Letnikov fractional derivatives coincide with the Marchaud fractional derivatives (see Section 20.3 in $[1,2]$ ) for the functions from space $L_{r}(\mathbb{R})$, where $1 \leqslant r<\infty$ (see Theorem 20.4 in $[1,2])$. Moreover both the Grünwald-Letnikov and Marchaud derivatives have the same domain of definition. The Marchaud fractional derivative is defined by the equation

$$
{ }^{M} D_{x_{j}}^{\alpha, \pm} f(\mathbf{r}, t)=\frac{1}{a(\alpha, s)} \int_{0}^{\infty} \frac{\Delta_{z_{j}}^{s, \pm} f(\mathbf{r}, t)}{z_{j}^{\alpha+1}} d z_{j}, \quad(0<\alpha<s),
$$

where $\Delta_{z_{j}}^{s, \pm}$ is the finite difference of integer order $s$,

$$
\Delta_{z_{j}}^{s, \pm} f(\mathbf{r}, t)=\sum_{k=0}^{s} \frac{(-1)^{k} s !}{(s-k) ! k !} f\left(\mathbf{r}-k z_{j} \mathbf{e}_{j}, t\right),
$$

and $a(\alpha, s)$ is

$$
a(\alpha, s)=\frac{s}{\alpha} \int_{0}^{1} \frac{(1-\xi)^{s-1}}{(\ln (1 / \xi))^{\alpha}} d \xi .
$$

Using (5), we can note that derivatives (18) for integer orders $\alpha=n \in \mathbb{N}$ have the forms

$$
\mathrm{GL}_{\mathbb{D}_{C}^{+}}^{+}\left[\begin{array}{c}
n \\
j
\end{array}\right]=\frac{1 \pm(-1)^{n}}{2} \frac{\partial^{n}}{\partial x_{j}^{n}} .
$$

Therefore the continuum fractional derivatives ${ }^{\mathrm{GL}} \mathbb{D}_{C}^{+}\left[\begin{array}{c}n \\ j\end{array}\right]$ are the usual derivatives of integer order $n$ for even values $\alpha$ only, and the continuum operators ${ }^{\mathrm{GL}} \mathbb{D}_{C}^{-}\left[\begin{array}{l}n \\ j\end{array}\right]$ are the derivatives of integer order $n$ for odd values $\alpha$ only.

For bounded lattices, the fractional-order difference operators ${ }_{B}^{G L} \mathbb{D}_{L}^{ \pm}\left[\begin{array}{c}\alpha_{j} \\ j\end{array}\right]$ defined by (10) are transformed by the continuous limit

$$
\lim _{a_{j} \rightarrow 0+}\left({ }_{B}^{\mathrm{GL}} \mathbb{D}_{L}^{ \pm}\left[\begin{array}{c}
\alpha_{j} \\
j
\end{array}\right] f(\mathbf{m}, t)\right)={ }_{B}^{\mathrm{GL}} \mathbb{D}_{C}^{ \pm}\left[\begin{array}{c}
\alpha_{j} \\
j
\end{array}\right] f(\mathbf{r}, t),
$$

into the continuum fractional derivatives of the GrünwaldLetnikov type with respect to space coordinate $x_{j}$,

$$
{ }_{B}^{\mathrm{GL}} \mathbb{D}_{C}^{ \pm}\left[\begin{array}{c}
\alpha \\
j
\end{array}\right]=\frac{1}{2}\left({ }_{x_{j}^{1}}^{\mathrm{GL}} D_{x_{j},+}^{\alpha}{ }_{x_{j}^{2}}^{\mathrm{GL}} D_{x_{j},-}^{\alpha}\right)
$$

which contain the Grünwald-Letnikov fractional operators defined on the finite interval $\left[x_{j}^{1}, x_{j}^{2}\right]$ with $x_{j}^{1}=m_{j}^{1} a_{j}$ and $x_{j}^{1}=m_{j}^{2} a_{j}$, in the form

$$
\begin{aligned}
{ }_{B}^{\mathrm{GL}} D_{x_{j}, \pm}^{\alpha} f(\mathbf{r}, t)=\lim _{a_{j} \rightarrow 0+} \frac{1}{\left|a_{j}\right|^{\alpha}} \sum_{m_{j}=0}^{M_{j}^{ \pm}} & \frac{(-1)^{m_{j}} \Gamma(\alpha+1)}{\Gamma\left(m_{j}+1\right) \Gamma\left(\alpha-m_{j}+1\right)} \\
& \times f\left(\mathbf{r} \mp m_{j} \mathbf{a}_{j}, t\right),
\end{aligned}
$$


where

$$
M_{j}^{+}=\left[\frac{x_{j}-x_{j}^{1}}{a_{j}}\right], \quad M_{j}^{-}=\left[\frac{x_{j}^{2}-x_{j}}{a_{j}}\right] .
$$

The suggested forms of continuum fractional derivatives of the Grünwald-Letnikov type allow us to consider diffusion processes on bounded areas of nonlocal continuum.

The lattice diffusion equation (13) in the continuum limit gives the fractional diffusion equation with derivatives of noninteger orders with respect to space coordinates. This space-fractional diffusion equation for the probability density $f(\mathbf{r}, t)$ has the form

$$
\begin{aligned}
\frac{\partial f(\mathbf{r}, t)}{\partial t}= & -\sum_{i=1}^{3} D_{i}(\alpha)^{\mathrm{GL}} \mathbb{D}_{C}^{-}\left[\begin{array}{c}
\alpha_{i} \\
i
\end{array}\right] f(\mathbf{r}, t) \\
& +\frac{1}{2} \sum_{i=1}^{3} \sum_{j=1}^{3} D_{i j}(\alpha, \beta){ }^{\mathrm{GL}} \mathbb{D}_{C}^{-,-}\left[\begin{array}{cc}
\alpha_{i} & \beta_{j} \\
i & j
\end{array}\right] f(\mathbf{r}, t),
\end{aligned}
$$

where $D_{i}(\alpha)$ is the drift vector and $D_{i j}(\alpha, \beta)$ is the diffusion tensor for the continuum that are defined by the lattice coupling constants $g_{i}$ and $g_{i j}$ by the relations $D_{i}(\alpha)=$ $g_{i}, D_{i j}(\alpha, \beta)=2 g_{i j}$. Similarly, the diffusion equation for bounded lattice gives the fractional diffusion equation for bounded region of continua

$$
\begin{aligned}
\frac{\partial f(\mathbf{r}, t)}{\partial t}= & -\sum_{i=1}^{3} D_{i}(\alpha){ }_{B}^{\mathrm{GL}} \mathbb{D}_{C}^{-}\left[\begin{array}{c}
\alpha_{i} \\
i
\end{array}\right] f(\mathbf{r}, t) \\
& +\frac{1}{2} \sum_{i=1}^{3} \sum_{j=1}^{3} D_{i j}(\alpha, \beta){ }_{B}^{\mathrm{GL}} \mathbb{D}_{C}^{-,-}\left[\begin{array}{cc}
\alpha_{i} & \beta_{j} \\
i & j
\end{array}\right] f(\mathbf{r}, t) .
\end{aligned}
$$

It should be noted that coincidence of orders of fractional derivatives in the first and second terms allows us to represent the fractional diffusion equation (28) in the form of the spacefractional continuity equation

$$
\frac{\partial f(\mathbf{r}, t)}{\partial t}=-\sum_{i=1}^{3} \mathrm{GL}_{\mathbb{D}_{C}^{-}}^{-}\left[\begin{array}{c}
\alpha_{i} \\
i
\end{array}\right] J_{i}(\mathbf{r}, t),
$$

where $J_{i}$ is the probability flow

$$
J_{i}(\mathbf{r}, t)=D_{i}(\alpha) f(\mathbf{r}, t)-\frac{1}{2} \sum_{j=1}^{3} D_{i j}(\alpha, \beta){ }^{\mathrm{GL}} \mathbb{D}_{C}^{-}\left[\begin{array}{c}
\beta_{j} \\
j
\end{array}\right] f(\mathbf{r}, t) .
$$

Equation (31) can be considered as the fractional phenomenological Fick first law for nonlocal media. If $\alpha_{i}=1$ for all $i=1,2,3$, the continuity equation (30) can be represented as the standard form of the well-known continuity equation

$$
\frac{\partial f(\mathbf{r}, t)}{\partial t}=-\sum_{i=1}^{3} \frac{\partial J_{i}(\mathbf{r}, t)}{\partial x_{i}}
$$

where $J_{i}(\mathbf{r}, t)$ is defined by (31) with $\beta_{j} \neq 1$ in general.
For one-dimensional case with $D_{i}(\alpha)=0$ and $f(\mathbf{r}, t)=$ $f(x, t),(28)$ can be represented in the form

$$
\frac{\partial f(x, t)}{\partial t}=K(\mu) \nabla^{\mu} f(x, t)
$$

where $K(\mu)$ is the generalized diffusion constant,

$$
K(\mu)=\frac{1}{2} D_{11}(\alpha, \beta)
$$

and $\nabla^{\mu}$ is the fractional derivative of $\operatorname{order} \mu$,

$$
\begin{array}{r}
\nabla^{\mu}={ }^{\mathrm{GL}} \mathbb{D}_{C}^{-}\left[\begin{array}{c}
\alpha_{1} \\
x
\end{array}\right]{ }^{\mathrm{GL}} \mathbb{D}_{C}^{-}\left[\begin{array}{c}
\beta_{1} \\
x
\end{array}\right]={ }^{\mathrm{GL}} \mathbb{D}_{C}^{-}\left[\begin{array}{c}
\alpha_{1}+\beta_{1} \\
x
\end{array}\right], \\
\mu=\alpha_{1}+\beta_{1} .
\end{array}
$$

Here we use the semigroup property of fractional derivatives of the Grünwald-Letnikov type. Equation (33) describes the fractional diffusion processes with the Poissonian waiting time and the Lévy distribution for the jump length (see Section 3.5 of [22]). In [22] the space-fractional diffusion equation (33) contains the Weyl fractional derivative $\nabla^{\mu}$ of order $\mu$, of one-dimensional case. The solution of (33) can be obtained analytically by using the Fox function $H_{2,2}^{1,1}$ (for details see Section 3.5 of [22] and [28]). The exact calculation of fractional moments [22] gives

$$
\left\langle|x(t)|^{\delta}\right\rangle=\frac{2(K(\mu))^{\delta / \mu} \Gamma(-\delta / \mu) \Gamma(1+\delta)}{\mu \Gamma(-\delta / 2) \Gamma(1+\delta / 2)} t^{\delta / \mu},
$$

where $0<\delta<\mu \leq 2$.

Using (2), it is possible to demonstrate that the spacefractional diffusion equations are connected with continuous time random walk processes with diverging second moment of the jump length distribution [22].

If $\alpha_{j}=\beta_{j}=1$ for all $j=1,2,3$, then (28) and (29) give the well-known second-order diffusion equation

$$
\frac{\partial f(\mathbf{r}, t)}{\partial t}=-\sum_{i=1}^{3} D_{i} \frac{\partial f(\mathbf{r}, t)}{\partial x_{i}}+\frac{1}{2} \sum_{i=1}^{3} \sum_{j=1}^{3} D_{i j} \frac{\partial^{2} f(\mathbf{r}, t)}{\partial x_{i} \partial x_{j}},
$$

where $D_{i}=D_{i}(1)$ is the drift vector and $D_{i j}=D_{i j}(1,1)$ is the diffusion tensor for local continuum.

\section{Conclusion}

Lattice analog of the fractional-order differential equations for bounded and unbounded three-dimensional lattices with long-range jumps of particles is suggested. These lattice equations can be considered as a new microscopic basis to describe the fractional diffusion in nonlocal continua. In the lattice diffusion equations, we use the fractionalorder difference analogs of fractional derivatives, which are represented by kernels that describe long-range jumps of lattice particles. The proposed kernels of long-range jumps on the lattice can be considered for integer and fractional orders of suggested difference operators. The continuous limits for these diffusion equations with fractional-order differences 
give the continuum fractional derivatives of the GrünwaldLetnikov type with respect to space coordinates. The obtained fractional diffusion on nonlocal continua can be considered as a continuous limit of the suggested lattice diffusion, where the sizes of continuum elements are much larger than the distances between sites of the lattice. The main advantage of the suggested approach is a possibility to consider fractionalorder difference diffusion equations as tools for formulation of a microstructural basic model of fractional diffusion in nonlocal continua. The proposed three-dimensional lattice diffusion equations can play an important role in formulating discrete models of nonlocal processes in microscale and nanoscale.

It is interesting to generalize the suggested lattice approach to consider lattice Lévy flights subject to external force fields and the Galilean invariance. This transport process on lattice can be described by lattice fractional diffusion equations. We assume that the lattice Lévy flights in a constant force field are similar to lattice fractional diffusion in a constant velocity field by analogy with diffusion processes in continuum models [29].

We assume that the proposed lattice approach to the lattice fractional diffusion can be generalized to different types of Bravais lattices such as monoclinic, triclinic, hexagonal, and rhombohedral lattices. We also assume that the suggested approach to the fractional diffusion can be generalized for lattice models with the fractal spatial dispersion, which are suggested in [30] (see also [31, 32]), and the continuum limits of these fractal lattice models can give continuum models of fractal media $[33,34]$ that are described by non-integerdimensional space approach $[35,36]$.

\section{Conflict of Interests}

The author declares that there is no conflict of interests regarding the publication of this paper.

\section{References}

[1] S. G. Samko, A. A. Kilbas, and O. I. Marichev, Integrals and Derivatives of Fractional Order and Applications, Nauka i Tehnika, Minsk, Belarus, 1987.

[2] S. G. Samko, A. A. Kilbas, and O. I. Marichev, Fractional Integrals and Derivatives Theory and Application, Gordon and Breach, New York, NY, USA, 1993.

[3] A. A. Kilbas, H. M. Srivastava, and J. J. Trujillo, Theory and Applications of Fractional Differential Equations, vol. 204 of North-Holland Mathematics Studies, Elsevier Science B.V., Amsterdam, The Netherlands, 2006.

[4] F. Mainardi, "Fractional calculus: some basic problems in continuum and statistical mechanics," in Fractals and Fractional Calculus in Continuum Mechanics, A. Carpinteri and F. Mainardi, Eds., pp. 291-348, Springer, New York, NY, USA, 1997.

[5] D. Valério, J. J. Trujillo, M. Rivero, J. A. T. Machado, and D. Baleanu, "Fractional calculus: a survey of useful formulas," The European Physical Journal Special Topics, vol. 222, no. 8, pp. 1827-1846, 2013.

[6] F. Mainardi, Fractional Calculus and Waves in Linear Viscoelasticity, An Introduction to Mathematical Models, World Scientific, Singapore, 2010.
[7] V. E. Tarasov, Fractional Dynamics: Applications of Fractional Calculus to Dynamics of Particles, Fields and Media, Springer, New York, NY, USA, 2011.

[8] J. Klafter, S. C. Lim, and R. Metzler, Eds., Fractional Dynamics. Recent Advances, World Scientific, Singapore, 2011.

[9] M. M. Meerschaert and A. Sikorskii, Stochastic Models for Fractional Calculus, vol. 43 of de Gruyter Studies in Mathematics, Walter de Gruyter \& Co., Berlin, Germany, 2012.

[10] V. Uchaikin and R. Sibatov, Fractional Kinetics in Solids: Anomalous Charge Transport in Semiconductors, Dielectrics and Nanosystems, World Scientific, Singapore, 7th edition, 2013.

[11] V. E. Tarasov, "Continuous limit of discrete systems with longrange interaction," Journal of Physics, A: Mathematical and General, vol. 39, no. 48, pp. 14895-14910, 2006.

[12] V. E. Tarasov, "Map of discrete system into continuous," Journal of Mathematical Physics, vol. 47, no. 9, Article ID 092901, 2006.

[13] V. E. Tarasov, "Lattice model with power-law spatial dispersion for fractional elasticity," Central European Journal of Physics, vol. 11, no. 11, pp. 1580-1588, 2013.

[14] V. E. Tarasov, "Fractional gradient elasticity from spatial dispersion law," ISRN Condensed Matter Physics, vol. 2014, Article ID 794097, 13 pages, 2014.

[15] V. E. Tarasov, "Lattice with long-range interaction of power-law type for fractional non-local elasticity," International Journal of Solids and Structures, vol. 51, no. 15-16, pp. 2900-2907, 2014.

[16] A. K. Grünwald, "About "limited" derivations their application," Journal of Applied Mathematics and Physics, vol. 12, pp. 441-480, 1897 (German).

[17] A. V. Letnikov, "Theory of differentiation with arbitrary pointer," Matematicheskii Sbornik, vol. 3, pp. 1-68, 1868 (Russian).

[18] I. Podlubny, Fractional Differential Equations: An Introduction to Fractional Derivatives, Fractional Differential Equations, to Methods of Their Solution and Some of Their Applications, Academic Press, San Diego, Calif, USA, 1998.

[19] V. E. Tarasov, "Lattice model of fractional gradient and integral elasticity: long-range interaction of Grünwald-Letnikov-Riesz type," Mechanics of Materials, vol. 70, no. 1, pp. 106-114, 2014.

[20] V. E. Tarasov, “Toward lattice fractional vector calculus," Journal of Physics, A: Mathematical and Theoretical, vol. 47, no. 35, Article ID 355204, 2014.

[21] J. P. Bouchaud and A. Georges, "Anomalous diffusion in disordered media: statistical mechanisms, models and physical applications," Physics Reports, vol. 195, no. 4-5, pp. 127-293, 1990.

[22] R. Metzler and J. Klafter, "The random walk's guide to anomalous diffusion: a fractional dynamics approach," Physics Reports, vol. 339, no. 1, pp. 1-77, 2000.

[23] G. M. Zaslavsky, "Fractional kinetic equation for Hamiltonian chaos," Physica D: Nonlinear Phenomena, vol. 76, no. 1-3, pp. 110-122, 1994.

[24] A. I. Saichev and G. M. Zaslavsky, "Fractional kinetic equations: solutions and applications," Chaos, vol. 7, no. 4, pp. 753-764, 1997.

[25] R. Metzler and T. F. Nonnenmacher, "Space- and timefractional diffusion and wave equations, fractional FokkerPlanck equations, and physical motivation," Chemical Physics, vol. 284, no. 1-2, pp. 67-90, 2002.

[26] G. M. Zaslavsky, "Chaos, fractional kinetics, and anomalous transport," Physics Reports: A Review Section of Physics Letters, vol. 371, no. 6, pp. 461-580, 2002. 
[27] V. E. Tarasov and G. M. Zaslavsky, "Fokker-Planck equation with fractional coordinate derivatives," Physica A: Statistical Mechanics and Its Applications, vol. 387, no. 26, pp. 6505-6512, 2008.

[28] F. Mainardi, G. Pagnini, and R. K. Saxena, "Fox $H$ functions in fractional diffusion," Journal of Computational and Applied Mathematics, vol. 178, no. 1-2, pp. 321-331, 2005.

[29] S. Jespersen, R. Metzler, and H. C. Fogedby, "Lévy flights in external force fields: langevin and fractional Fokker-Planck equations and their solutions," Physical Review E-Statistical Physics, Plasmas, Fluids, and Related Interdisciplinary Topics, vol. 59, no. 3, pp. 2736-2745, 1999.

[30] V. E. Tarasov, "Chains with the fractal dispersion law," Journal of Physics A: Mathematical and Theoretical, vol. 41, no. 3, Article ID 035101, 2008.

[31] T. M. Michelitsch, G. A. Maugin, F. C. Nicolleau, A. F. Nowakowski, and S. Derogar, "Dispersion relations and wave operators in self-similar quasicontinuous linear chains," Physical Review E: Statistical, Nonlinear, and Soft Matter Physics, vol. 80, no. 1, Article ID 011135, 8 pages, 2009.

[32] T. M. Michelitsch, G. A. Maugin, F. C. G. A. Nicolleau, A. F. Nowakowski, and S. Derogar, "Wave propagation in quasicontinuous linear chains with self-similar harmonic interactions: towards a fractal mechanics," in Mechanics of Generalized Continua: Advanced Structured Materials, vol. 7, pp. 231-244, 2011.

[33] B. O’Shaughnessy and I. Procaccia, "Analytical solutions for diffusion on fractal objects," Physical Review Letters, vol. 54, no. 5, pp. 455-458, 1985.

[34] R. Metzler, W. G. Glöckle, and T. F. Nonnenmacher, "Fractional model equation for anomalous diffusion," Physica A: Statistical Mechanics and Its Applications, vol. 211, no. 1, pp. 13-24, 1994.

[35] V. E. Tarasov, "Vector calculus in non-integer dimensional space and its applications to fractal media," Communications in Nonlinear Science and Numerical Simulation, vol. 20, no. 2, pp. 360-374, 2015.

[36] V. E. Tarasov, "Anisotropic fractal media by vector calculus in non-integer dimensional space," Journal of Mathematical Physics, vol. 55, no. 8, Article ID 083510, 2014. 

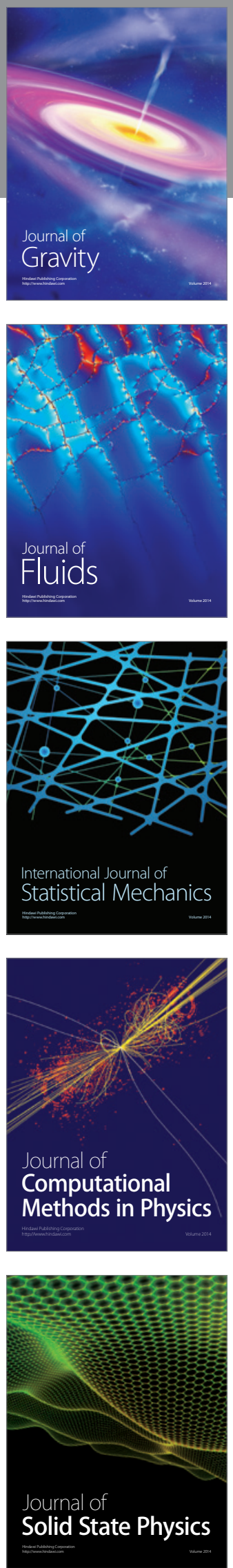

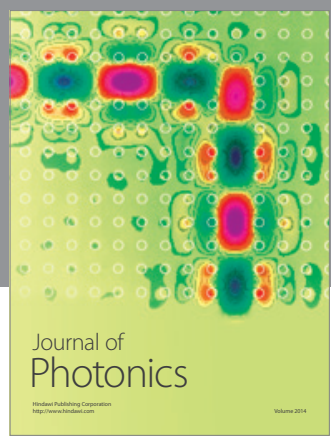

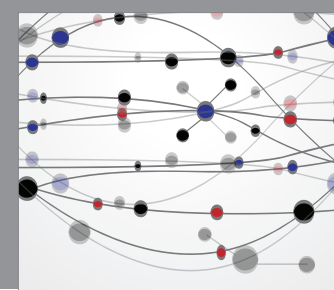

The Scientific World Journal

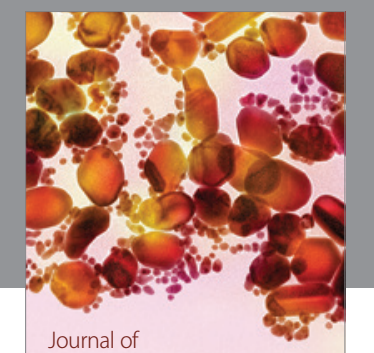

Soft Matter
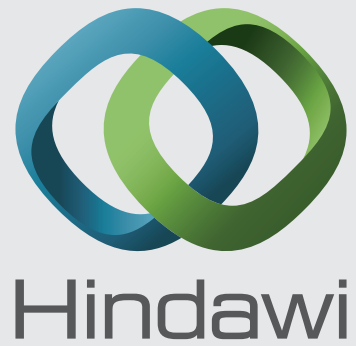

Submit your manuscripts at

http://www.hindawi.com
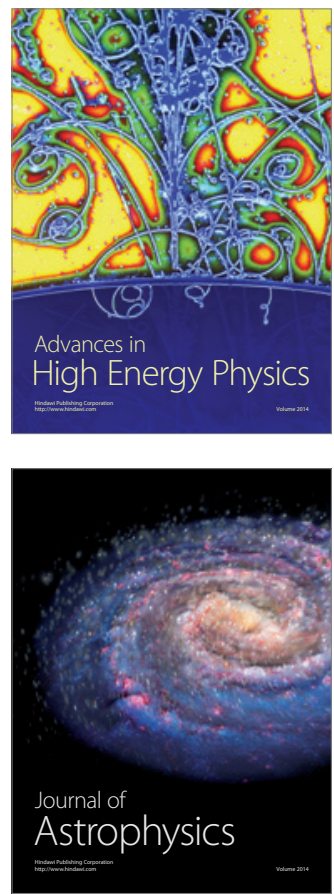
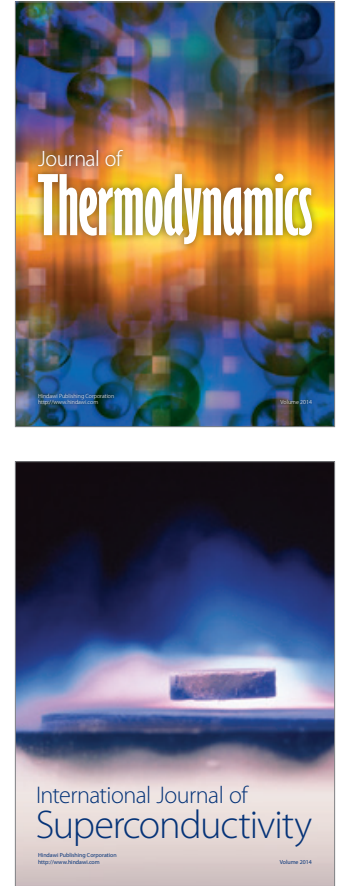
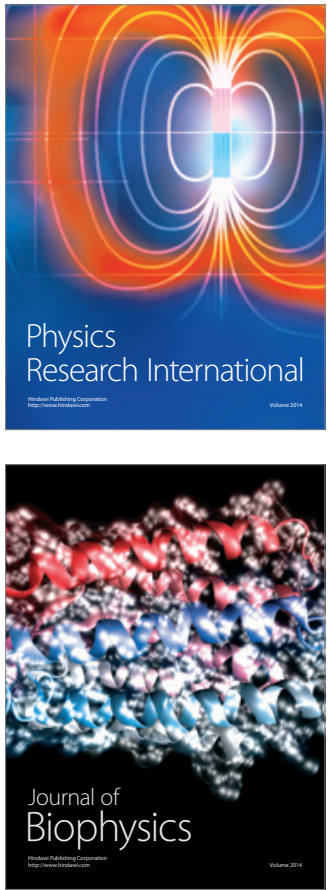
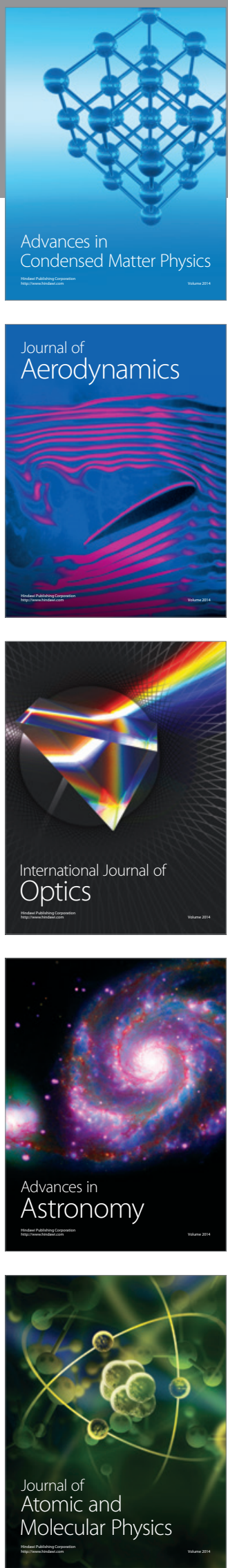